\title{
La materialidad de ficción y el tercer tiempo del objeto arqueológico- histórico (Siglos XIX-XX)
}

\author{
Fiction materiality and the third time of the historical-archaeological object (XIX-XX \\ Century)
}

Francisco Rivera ${ }^{\mathrm{i}}$, Rodrigo Lorca ${ }^{\mathrm{i}}$ Felipe Rojas Toro ${ }^{\mathrm{ii}}$

\section{RESUMEN}

A fines del siglo $X I X$ y principios del siglo $X X$, constatamos que junto a la nueva industria que se desarrolla con la naciente gran minería, la literatura chilena exhibe una presencia significativa de objetos domésticos e industriales. Considerando esta presencia de los objetos en los textos de ficción, este artículo explora el rol de ellos en la literatura para luego discutir su exposición como obras de arte y su conservación como vestigios arqueológicos. Se propone reflexionar sobre el rol de la materialidad arqueológica-histórica y la construcción de la memoria a través de tres diferentes formas de tiempo (literario, artístico y arqueológico), planteando que la representación de la materialidad no es sólo un hecho literario y artístico, sino también un fenómeno cultural que abarca contextos sociales, históricos y económicos de interesante perspectiva para una arqueología del pasado reciente. Objetos reales o imaginarios, vestigios físicos o representaciones, por medio de una lectura arqueológica buscamos examinar y evaluar el efecto que la materialidad ejerce sobre la sociedad que los describe, transforma, expone y conserva, en una época de especial relevancia histórica: la expansión capitalista ocurrida en Chile a fines del siglo XIX y principios del siglo $\mathrm{XX}$.

Palabras Claves: Materialidad, Literatura,Arte,Tiempo, Arqueología Histórica

\begin{abstract}
At the end of the 19th century and early 20th century, along with the new emerging mining industry, we notice that Chilean literature exhibits a significant presence of domestic and industrial objects. This article explores the role of them in literature to discuss afterwards their exposure as works of art and finally as archaeological remains. In this perspective, we seek to discuss on the role of historical-archaeological materiality and memory structure process through three different forms of time (literary, artistic and archaeological).We propose that their representation is not only a literary and artistic fact, but also a cultural phenomenon that encompasses social, historical and economic contexts of stimulating perspective for the archaeology of the contemporary past. Real or imaginary objects, physical remains or representations, by an archaeological reading we seek to examine their significance and evaluate the outcome that materiality encompasses on the society that describes, transforms, exhibits and preserves them in a period of singular historical significance: that is, capitalist expansion that took place in Chile at the end of the 19th and early 20th century.
\end{abstract}

Key Words: Materiality, Literature, Art, Time, Historical Archaeology

i SurAndino Estudios Arqueológicos y Patrimoniales Ltda. Correo-e:f.riveraamaro@gmail.com; rlorcah@gmail.com

ii Syna Bern/Gewerkschaft, Suiza. Correo-e: rojastoro1@gmail.com

Recibido: 21 abril, 2014. Revisado: 10 octubre, 2014. Aceptado: 30 noviembre, 2014. 
(...) aquello de perseguir objetos en los que no creemos, ni queremos, ni podemos radicar el énfasis de vivir (...); inocentemente hacemos del recuerdo un conocimiento del más allá adosal

(de Rokha, 1954: 505).

\section{INTRODUCCIÓN}

En uno de los pasajes de su novela Casa Grande, el escritor chileno Luis Orrego Luco retrata una escena de la vida de la clase aristocrática en Chile de principios del siglo $X X$, a través de la descripción de los objetos que atiborran el salón de su personaje Olga Sánchez,

"Sobre mesita rodeada de tazas de porcelana japonesa de estraños dibujos azúl y rosa, encontrábase lista la gran tetera de plaqué con lámpara de alcohol, en la cual se preparaba el samovar, como decia, empleando la palabra rusa, con el prurito de extrangerismo de nuestro mundo de tono, tan aficionado árefrescar sus aires en la colonia cosmopolita de Paris. Las paredes, tapizadas con riquísimo papel Luis XV, de listas de plata y verde nilo, en admirable imitación de razo de seda, casi desaparecían cubiertas por grabados con marcos de laca blanca y asuntos de Fragonard y de Wattean, acuarelas de Villegas y Pradilla, un cuadro de Urjell, platos de porcelana, entre los cuales algunos de mérito, varias de las enormes peinetas llamadas de teja, de carei, con fantásticas cinceladuras y dibujos, de esas usadas por las abuelas del siglo XVIII. La cajuela de madera tallada, indicaba pasión naciente por objetos antiguos" (Orrego Luco, 1908:185186).

Este artículo tiene por objetivo proponer una aproximación teórica a los tiempos del objeto histórico.Tomando como ejemplo algunas referencias del realismo literario chileno, discutiremos tres tiempos de esta materialidad: literario, artístico y arqueológico. Consideramos como problema de estudio que su representación, como se observa en la descripción hecha por Orrego Luco en el párrafo anterior, no es sólo un hecho literario o la descripción de simples adornos dentro de una escena y una narrativa en particular, sino que refleja un fenómeno cultural que abarca contextos sociales, históricos y económicos, de interesante perspectiva de análisis para la arqueología histórica. Si bien para la arqueología actual no es novedoso utilizar a la literatura como fuente de datos' (Orser 2000; Schávelzon 2002, entre otros), tampoco lo es para los análisis literarios que se interesan a la cultura material descrita en los textos de ficción (Brown, 2003, 2004; Caraion, 2005, 2007). Debido a ello, nuestra intención en este artículo no se reduce a una mera confrontación de referencias literarias y muestras materiales. Por supuesto, tampoco desmerecemos las aproximaciones arqueológicas tradicional es las cuales describirían aquellos objetos, categorizándolos e insertándolos en un tiempo histórico predeterminado, para así elaborar interpretaciones coherentes según su contexto ${ }^{2}$. Sin embargo, proponemos aquí otro ejercicio.Asumiendo las dificultades con las que se enfrentan las lecturas habituales sobre el efecto que los objetos produjeron tanto en el pasado como hoy en día, pretendemos aportar con otra arista a la discusión epistemológica de la materialidad del pasado contemporáneo o reciente (Buchli y Lucas, 200 I;Brown, 2004; GonzálezRuibal, 2006; Harrison y Schofield, 2010; Olsen et al. 20I2; Olsen, 20I3; Pétursdóttir, 20I2, 20I3). Y para eso creemos que una mirada a las representaciones literarias de una serie de objetos cotidianos 0 industriales nos puede entregar una visión fresca sobre el rol de la materialidad y la relación sujetoobjeto de un momento histórico particular, como lo fue la expansión capitalista ocurrida en Chile a fines del siglo XIX y principios del siglo XX.

En un sentido amplio, reflexionaremos sobre el rol de la materialidad arqueológica-histórica y la construcción de la memoria a través de tres diferentes formas de tiempo, que entendemos como diferentes formas de transformación conceptual del objeto. Esto, con el fin último de abrir una futura discusión sobre la relación, que entendemos como dinámica y versátil, de las modalidades materiales con sus espacios de exhibición; museos, exposiciones y/o catálogos. Esta propuesta de análisis de los objetos históricos que entendemos como categorizados en vestigios arqueológicos ${ }^{3}$ y como representaciones, nos permite finalmente considerar interesantes elementos teóricos para comprender los alcances de las políticas de patrimonialización de los bienes culturales asociados al pasado contemporáneo o reciente. 


\section{OBJETO LITERARIO, OBJETO DE CONSUMO}

Dentro de la literatura chilena de fines del siglo XIX y principios del siglo $\mathrm{XX}$, constatamos una importante cantidad de referencias de objetos. En varios textos, la materialidad se describe y se expone no solamente como elementos que detallan y sitúan el ambiente y el contexto de los personajes, sino también como agentes importantes en el entramado de sus historias. En esta primera parte tomaremos algunos ejemplos para nuestro punto de partida ${ }^{4}$, el cual será entender que la literatura, como una acción comunicativa a través de la cual se reproducen las estructuras simbólicas de la cultura (Habermas, 1984), expone lo que proponemos en este artículo como un primer tiempo del objeto arqueológico-histórico. Es decir, éste tiene como función principal en los textos de ficción la de construir un universo referencial (Caraion, 2007), pero también la de constituir las relaciones entre los individuos. Siguiendo a Caraion (2007), los personajes en los textos de ficción no solamente integran genealogías o revelan historias, sino que también adquieren, guardan, compran e intercambian objetos. Continuemos con la descripción que hace Orrego Luco del salón antes mencionado,

"En el rincón había también vitrina de madera de rosa incrustada y cincelados bronces, detrás de cuyos cristales, redondeados y salientes, se ocultaban objetos de marfil; abanicos de cabritilla pintada, con estrecho pais y ancho varillaje del tiempo de Goya y de María Luisa; monitos de porcelana de Sajonia (n.d.a.: ver Figura I); tazas de Sévres con marca de fábrica y la especial del servicio del Rey; pocillos españoles, dorados por dentro; vieja loza de Talavera; porcelanas de Capo-di-Monti y una tacita lejítima con relieves de danza griega, de Wegwood, que valía por todos los objetos allí encerrados en obedecimiento á los preceptos siempre tiránicos de la moda que aconsejaba la vuelta á lo antiguo" (Orrego Luco, 1908: I85I86).

Todo este amplio conjunto de objetos no sólo representan el medio social, el nivel socio-cultural y económico, sino que también y en forma más íntima, buscan mostrar ciertos rasgos de carácter, deseos y emociones de los personajes (Caraion, 2007). En ese sentido, la literatura nos da pie para entender

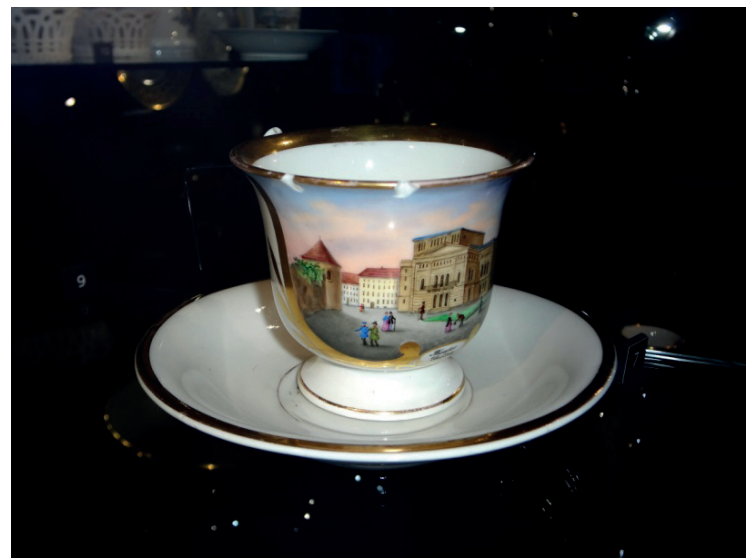

Figura 1: Porcelana de Meissen, Alemania (1854). Museo de Artes Decorativas, Santiago, Chile (Foto: Rodrigo Lorca)

Figure 1: Meissen porcelain, Germany (1854). Museum of Decorative Arts, Santiago, Chile (Photography: Rodrigo Lorca).

la idea central de este artículo: toda práctica social estará siempre mediatizada por los objetos, lo que nos obliga necesariamente a destacar las relaciones complejas que se establecen entre los individuos y la materialidad, en una dinámica que confiere un lugar preponderante a las prácticas sociales más allá de su función mediadora entre la acción y el individuo (Olsen et al., 2012; Olsen 20I3).

Como lo vimos en la cita anterior de Orrego Luco, la materialidad se presenta entonces como un agente protagonista de las historias que se entretejen alrededor de los personajes literarios. Objeto e individuo aparecen así en la literatura estrechamente entrelazados, actuando los primeros como un vínculo entre la persona y su medio. La materialidad proyecta así una imagen y delinea un ambiente particular alrededor de su propietario, el cual es reconocido por los otros actores sociales muchas veces de forma inconsciente. Es a partir de este reconocimiento que tenemos indicios de la relación de la materialidad con la sociedad que los produjo, como un espacio social el cual constituye y es constituido por los individuos, o en este caso, por los personajes de ficción. Siguiendo a Bourdieu (1998: 75),

"si resulta posible leer todo el estilo de vida de un grupo en el estilo de su mobiliario y de su forma de vestir, no es solamente porque estas propiedades sean la objetivación de las necesidades económicas y culturales que han 
determinado su selección; es también porque las relaciones sociales objetivadas en los objetos familiares, en su lujo o en su pobreza, (...) se imponen por mediación de unas experiencias corporales(...) profundamente inconscientes".

En el marco de una nueva sociedad de consumo surgida gracias a las ganancias de la naciente gran minería (salitre, carbón, plata o cobre) y en el cual las actividades humanas se vuelcan a la producción como respuesta de la Revolución Industrial europea, observamos este tipo de reconocimiento y las formas de exposición de esta nueva relación a través de las representaciones de los objetos que los autores plasman en sus obras. Los vínculos y reconocimiento de esta nueva burguesía nacional industrial se cristalizan entonces a través de esta nueva representación material (Caraion, 2005, 2007): de la mercancía (Marx, 1980) y del consumo (Baudrillard, 1968).

Si para Marx (1980), por un lado, el interés se centra en el carácter del fetiche de la mercancía como forma de objetividad y de su correspondiente comportamiento subjetivo, para Baudrillard (2009 [1974]) en cambio, el consumo del objeto será entendido como una estructura de comunicación. Para este último, el consumo es un sistema que asegura una moral, un sistema de valores y una estructura de intercambio. En éste, las personas adhieren inconscientemente a las reglas estructurales del sistema, por lo que la comunicación social puede tener lugar exclusivamente en la dirección establecida por el código de la diferencia (Baudrillard 2009, [1974]). Por ende, como un sistema de intercambio y producción de valores, para el autor el consumo constituye el lenguaje utilizado por la sociedad contemporánea como un mecanismo de manipulación de signos y significado, desmarcándose así de otros críticos del capitalismo que ven al consumidor como un sujeto pasivo frente a la publicidad (Baudrillard 2009 [1974]). El afiche publicitario de la Figura 2 refleja este cambio de perspectiva del consumo, que va de un sistema económico a un sistema de comunicación simbólica, el cual utiliza elementos como lo "refinado" o lo "extranjero" para resaltar el valor del objeto. Lo anterior nos permite así entender que el consumo se convierte en la estructura básica de la sociedad contemporánea y no es ya solamente un comportamiento económico puesto en negativo.

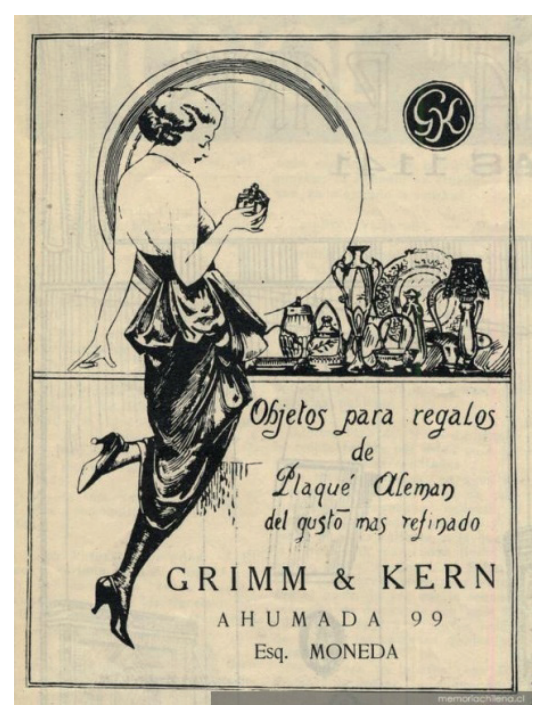

Figura 2: Publicidad de objetos de la marca Grimm \& Kern (Fuente: Memoria Chilena, disponible en <www.memoriachilena. $\mathrm{cl} / 602 / \mathrm{w} 3$-article-73712.html $>$ )

Figure 2: Advertising objects from Grimm \& Kern brand (Source: MemoriaChilena, available in $<w w w . m e m o r i a c h i l e n a . c l / 602 / w 3-$ article-73712.html >)

Así como Orrego Luco, Blest Gana nos expone también estas transformaciones y el sistema de comunicación simbólica desde la materialidad. El autor escribe,

Los nombres más notables de la industria parisiense estaban ahí representados. Cucho hizo observar un servicio de oro para postre, firmado 'Falize', cincelado como una obra destinada á un museo. Explicó el mérito singular de un servicio de te, para cuatro personas, de la más delicada porcelana de Sèvres, en el que el azucarero, la lechera y las cucharillas de plata proclamaban el arte eximio de la casa de Froment-Meurice. Las alhajas de Boucheron tenían una gran novedad de formas" (Blest Gana, 1904: 363).

Como se puede apreciar tanto de la cita anterior como del texto de Orrego Luco, la exuberancia de nuevos objetos le otorga legitimidad a una nueva clase social, como la nueva burguesía industrial, que carece de fundamentos históricos y culturales, y en la cual la apropiación de la materialidad aparece como una nueva inversión local. Así entonces, los objetos se instalan en la literatura representando las comodidades burguesas 
y sus aspiraciones. Blest Gana lo ilustra nuevamente cuando escribe,

"Sobre mesas angostas y largas, en cajas con tapa de vidrio fabricadas por la casa de Yansen, todas aquellas maravillas de costura y de arte formaban una exhibición primorosa. La corona de príncipe, bordada, cincelada ó pintada, según la naturaleza de los objetos, realzaba la riqueza de cada, al par que ofrecía una prueba de la pretenciosa prodigalidad con que los padres de la desposada querían presentarse á la alta sociedad, al mundo de sus anhelos" (Blest Gana, 1904: 320).

En este tipo de novelas observamos que los objetos juegan un rol fundamental en las relaciones entre los personajes, en la medida en que éstos últimos simultáneamente interpretan la materialidad y categorizan a sus propietarios. De esta manera, los objetos participan en la constitución de la visión del mundo social de los personajes, definiendo y permitiendo identificar una pertenencia de clase.

En este nuevo contexto de expansión capitalista que se visualiza desde fines del siglo $\mathrm{XIX}$, se observa entonces una nueva relación del individuo con el mundo material, a través del deseo de posesión de la materialidad. El paradigma del éxito social se modifica progresivamente, y la posesión de objetos se convierte casi en una condición sine qua non de la felicidad. Así entonces el deseo entendido como el sentimiento permanente de falta o ausencia, se presenta como el nuevo motor socio-económico que establece no solamente el tipo de vínculos a los objetos, sino que también la relación entre los individuos. Como bien lo plantea Caraion (2007), se trata de una relación que se instaura a partir de la necesidad creada por aquellos que poseen los objetos y la frustración creada por aquellos que los desean. En ese marco, observamos en una cita de Galeano (I984), nuevamente esta idea de legitimar por medio de la materialidad el deseo de una posición social determinada, en la cual hace referencia al sello de las botellas “Old Champaign Cognac"(Figura 3), y cuyo referente material encontramos durante las excavaciones de rescate en el marco de un proyecto de impacto ambiental en el norte de Chile (Rivera y Delgado, 2009),

“I832 (...) También en Chile los caballeros bailan y visten a la moda francesa, imitan a
Byron al anudarse la corbata, y en la mesa obedecen al cocinero francés; a la inglesa toman el té y a la francesa beben trago. Cuando Vicente Pérez Rosales instaló su fábrica de aguardiente, compró en Paris los mejores alambiques y una buena cantidad de etiquetas de dorados arabescos y finas letras que decían: Old Champaign Cognac. A la puerta de su despacho, hizo pintar un gran cartel: IMPORTACIÓN DIRECTA. El sabor no sería muy-muy, pero sería casi-casi; y nadie quedó con llagas en el estómago. El negocio marchaba a las mil maravillas y la fábrica no daba abasto, pero don Vicente sufrió un ataque de patriotismo y decidió que no podía seguir viviendo en estado de traición: Esta buena fama sólo a Chile corresponde. Arrojó al fuego las etiquetas europeas y su despacho estrenó otro cartel, más grande todavía: FÁBRICA NACIONAL. Las botellas lucen ahora un nuevo vestido: etiquetas impresas aquí, que dicen Coñac chileno. No se vende ni una" (Galeano, 1984: I30-I3I).

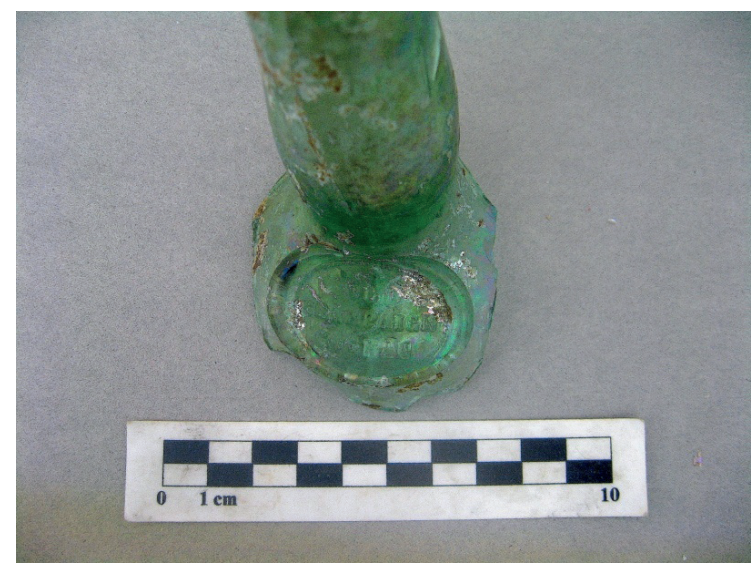

Figura 3: Fragmento de botella con sello "Old Champaign Cognac" (Rivera \& Delgado 2009) (Foto: Francisco Rivera) Figure 3: Fragment of bottle with seal "Old Champaign Cognac" (Rivera \& Delgado 2009) (Photography: Francisco Rivera).

Este rol de legitimación social y transmisión de valores por medio de la materialidad que observamos en el ejemplo anterior, nos recuerda aquel que Bourdieu puso ya en evidencia,

"no existe herencia material que no sea a la vez una herencia cultural, y los bienes familiares tienen como función no sólo la de dar testimonio fisico de la antigüedad y continuidad de la familia $y$, por ello, la de consagrar su identidad social, 
no disociable de la permanencia en el tiempo, sino también la de contribuir prácticamente a su reproducción moral, es decir, a la transmisión de los valores, virtudes y competencias que constituyen el fundamento de la legítima pertenencia a las dinastías burguesas (Bourdieu, 1998: 75).

Por un lado observamos esta exuberancia de la materialidad aristocrática, y el rol que ésta ejerce en la legitimización de una nueva clase social burguesa industrial. Sin embargo, también contamos con su opuesto: textos que ofrecen el detalle de aquellos pequeños objetos que forman parte del contexto de la precariedad y la ausencia. Baldomero Lillo lo expone así tomando como eje el mundo obrero-minero,

"ambos con la mano derecha sostenían la lámpara y con la izquierda un manojo de pequeños trozos de cordel en cuyas extremidades había atados un botón o una cuenta de vidrio de distintas formas y colores; eran los tantos o señales que los barreteros sujetan dentro de las carretillas de carbón para indicar arriba suprocedencia" (Lillo, I968: I42143).

Por otro lado, en el marco de esta exploración literaria que se ubica en ambos mundos real e imaginario, se exhiben también objetos de una sociedad que se transforma a un ritmo vertiginoso, donde el tema de la pérdida es particularmente elocuente. En un período de grandes transformaciones sociales, los textos se mueven en una dimensión más bien melancólica, situando paradojas entre lo moderno y lo tradicional, y ubicando a los objetos en el recuerdo como testigos de esplendores pasados, “(...)entre los pobres objetos guardados como reliquias: el mate y su bombilla, el sahumador de filigrana, la Virgen y el san José de bulto, que le formaban un rincón de consuelo, la evocación de otra existencia, en su muda esperanza de desterrados" (Blest Gana, 1904: 47I). Podemos ver que estos relatos se construyen con una visión casi foránea en términos temporales, describiendo vestigios de una sociedad que se diluye, en un lento proceso de olvido, el cual es necesario reconstruir desde el presente,

"A las once horas tres cuartos, el incendio se pronunció en el ángulo norte de los imponentes edificios de la Aduana. Iban allí a convertirse en cenizas (...) valores por millones de pesos en mercaderías de nacionales $i$ estranjeros. La porcelana, el mármol, el bronce, la plata, el hierro, el plomo, el papel, los cristales, las telas, todo iba a fundirse, a liquidarse, a formar como un río de ardiente lava, que al enfriarse lentamente por la acción del aire, iba a ofrecer una píngüe entrada a oscuros especuladores que concibieron la idea de pasearse por nuestro territorio, exhibiendo a la vista de los pueblos admirados los trozos de esa masa multiforme"(Vargas, 1870: 214).

Aquella "masa multiforme" relatada por Vargas,lo podemos entender en un sentido metafórico como una visión de tipo casi ornamental sobre la materialidad, como una forma de estetización de los objetos. En esta nuevas (multi)formas, los objetos se integran en nuevos campos narrativos, y observamos que se delinea una bipartición de la percepción de ellos; por un lado, los objetos del mundo doméstico aristocrático se muestran pretendidos y deseados, pero como productos generados por una industria que los produce en masa, carentes de humanidad y destinados al consumo empedernido de la nueva burguesía (Caraion, 2007). Por otro lado, el mundo laboral-industrial se representa con objetos admirables, fruto del trabajo fuertemente valorado de un obrero que termina formando parte también de la maquinaria que domina. Lillo lo comprende y expone de este modo aquellas fronteras difusas del individuo-máquina, robotizado por una industria fría y despersonalizada,

“El maquinista, al asir con la diestra el mango de acero del gobierno de la máquina, pasa instantáneamente a formar parte del enorme y complicado organismo de hierro. Su ser pensante conviértese en autómata. Su cerebro se paraliza. A la vista del cuadrante pintado de blanco, donde se mueve la aguja indicadora, el presente, el pasado y el porvenir son reemplazados por la idea fija. (...) Por eso sus pupilas, su rostro, su pensamiento se inmovilizan. Nada ve, nada oye de lo que pasa a su derredor, sino la aguja que gira y el martillo de señales que golpea encima de su cabeza" (Lillo, 1968: 262-263).

Habría que recordar que el siglo XIX es una época de inventos: la locomotora, el teléfono o el fonógrafo "fijan lo efímero, detienen el tiempo, 
acortan las distancias" (Caraion, 2005: I 2), y como lo podemos apreciar, también transforman y modifican las viejas formas de relacionarse con la materialidad. Como bien lo expone Caraion (2007), las máquinas se vuelven cada vez más sofisticadas y especializadas, utilizándose también para producir otras máquinas en un movimiento paradójico de autogeneración, diversificándose al mismo tiempo los accesorios y las cosas cotidianas. Podemos observar entonces que surge una imagen de poder material, asociando los objetos industriales a su capacidad, por un lado creadoras (de objetos cotidianos producidos en masa) pero también destructoras (de la individualidad y la humanidad). En este contexto, entendemos finalmente que el escritor no sólo plasma las nuevas modalidades de producción industrial, sino que también sitúa a todo este conjunto de nuevos objetos en diferentes campos del imaginario (Perec, 1965; Brown, 2003; Caraion, 2007).

\section{EL OBJETO EXPUESTO Y LA OBRA DE ARTE}

Considerando lo anteriormente expuesto, y reflexionando sobre el rol de esta construcción de diferentes campos del imaginario y de la ficción del objeto cotidiano e industrial, daremos aquí un salto. El proceso de expansión capitalista a través de la materialidad de ficción que hemos presentado, no trata solamente al número de objetos disponibles, sus modos de producción, uso y consumo, o su nivel de "connotación" (Baudrillard, 1968), sino que se instaura un nuevo proceso de creación de objetos nuevos igualmente "ficcionados". Creemos que esto se realiza a través de una nueva relación de las materialidades con nuevos espacios de exhibición; los museos, las exposiciones y los catálogos. Siguiendo a Faba, durante este período "los objetos e imágenes del pasado adquieren un valor de exhibición, circunscribiéndose de este modo un espacio que las resguarda, expone y promueve como testimonios del pasado" (Faba, 20 I 3: 29). Hemos visto que el proceso de industrialización ha permitido que los objetos se multipliquen, y dejen de ser únicos para ser de ahora en adelante siempre reproducibles. En ese marco, junto con el desarrollo económico derivado de la explotación de materias primas y los procesos de urbanización de los nuevos Estados nacionales, Chile incluido, nace en el siglo $X X$ un fenómeno de potenciación del objeto. Frente a la universalidad de las cosas presentes en la sociedad, se desarrollan estrategias de puestas en valor de la materialidad que buscan resaltar lo local. Nace lo que podríamos llamar una conservación patrimonial; una museología moderna (Shanks y Tilley, 1987; Heinich, 2009). Para Faba, "el museo constituye fundamentalmente un espacio consagrado a un sistema de objetos que aparece asociado a procesos de subjetivación propios de la industrialización y de la consolidación de la ciudad moderna" (Faba, 20I3: 23).

En ese escenario, se organizan en Chile algunos eventos históricos interesantes de este proceso, como por ejemplo la creación del Museo Histórico del Cerro Santa Lucía en 1874 y, fundamentalmente, la realización de la Exposición del Coloniaje de 1873 organizada por Benjamín Vicuña Mackenna, en aquellos años Intendente de Santiago (1872-1875) (Vicuña Mackenna 1873). Esta exposición abrió sus puertas al público el día miércoles 17 de septiembre en el Palacio de los Antiguos Generales (hoy Correo Central), en un intento por asemejar el evento a las grandes ferias internacionales de Europa y América del Norte. Esta exposición tenía por objetivo la exhibición del mundo material asociado al período colonial en Chile, montado a partir de las colecciones privadas pertenecientes a diversas familias aristocráticas del país. Muebles, joyas, trajes, monedas, pinturas, tapicerías, fusiles "con la pólvora de Chacabuco", “las espadas de los héroes" e incluso las actas originales de la fundación de Santiago firmada por Pedro de Valdivia (Vicuña Mackenna, 1873: 6), conformaron una plétora de objetos que invitaron a los visitantes a hacerse parte del orgullo nacional: “(...) aquí nos detenemos i dejamos vagar al curioso caballero i a la discreta dama entre los dos mil i tantos objetos reunidos en este que ayer era un eriazo al sol i hoy convida con sus galas a celebrar 'los buenos tiempos de Chile"' (Vicuña Mackenna, 1873: 7). Así también y como resultado de ambas actividades, durante el siglo XX la constitución definitiva del Museo Histórico Nacional en I9II (Alegría Licuime, 2007) y del Museo de Artes Decorativas en 1982, ambos en Santiago de Chile, nos interesan particularmente por los objetos que forman parte actualmente de sus colecciones (Figura I).

En este marco histórico de exposición del objeto, otro hito importante para discutir en nuestra propuesta tiene relación con la historia del arte contemporáneo. El desarrollo de la teoría del 
arte durante el siglo $\mathrm{XX}$ estuvo marcado sin lugar a dudas por la obra La Fuente, presentada por Marcel Duchamp en la exposición de la Sociedad de Artistas Independientes de 1917 en Nueva York. En ella observamos un fenómeno artístico particularmente interesante, ya observado en la Exposición del Coloniaje; no solamente las obras de arte sino que los objetos cotidianos comienzan a ser expuestos. Desde este momento, los objetos cotidianos son sacados de su contexto de uso para exhibirse. Si primero observamos un proceso en el cual la materialidad es desmitificada en la literatura, ahora también vemos uno en el cual esta es descontextualizada. Mientras algunos considerarán las obras de arte siguiendo lo que Heinich llama "un esquema de tautología", la cual viene a reducir un objeto a su materialidad espacial, otros asirán estos objetos según un "sistema de creencias", que consiste en aumentar el tema de los significados extrínsecos a su materialidad (Heinich 1993). Este punto nos parece de especial importancia, ya que presenta la idea que detrás de esta materialidad se plantea la idea de que objetos comunes de uso habitual, combinados o dispuestos de maneras diversas, se convirtieran en obras de arte por el simple deseo del artista. El objetivo principal de este nuevo concepto de ready-made fue demostrar la imposibilidad de establecer una definición única de lo que se entiende por arte, expresando así la ausencia de criterios universales que definieran lo que es de lo que no lo es (Dickie, 1969). Pero esto supuso variados y nuevos problemas: el principal, el de los criterios de definición de lo artístico (Carroll, 1995). En esta línea, el filósofo e historiador del arte Arthur Danto retoma el problema planteado acerca de la definición de lo que es arte a partir de la llamada "Brillo Box" de Andy Warhol (Danto, 1964, 198I). Para el autor, esta obra que consiste en dos grupos de cajas idénticas (el primer grupo fue hecho por el artista y el otro es un producto industrial de uso cotidiano), ejemplificaría el problema del arte; esto es, que no es posible definir el arte mediante criterios meramente perceptivos o, planteado en sus propios términos, que una obra de arte $y$ un objeto común pueden ser perceptivamente indiscernibles (Danto, 1981).

Con el fin de problematizar sobre el tiempo de las materialidades, buscaremos en la teoría de Danto un marco de referencia teórico para nuestra reflexión sobre las transformaciones de un tercer tiempo arqueológico. Planteamos que el problema presentado originalmente por Duchamp oWarhol, y retomado teóricamente por Danto, puede aplicarse al estudio de la materialidad de ficción como un nuevo tiempo del objeto, proponiendo que así como las obras de arte, lo que distingue a un vestigio de un objeto común no debiera basarse en criterios perceptivos, como su belleza, originalidad o incluso antigüedad, poniendo énfasis por el contrario en la trasformación categorial de los objetos como un nuevo tiempo arqueológico. Lo que buscamos discutir en este segundo apartado es lo que Danto propone y pretende encontrar, esto es, una propiedad no perceptible de las obras de arte; es decir, un elemento relacional definitorio que sirva como solución del problema de la demarcación.

Para Danto, una obra de arte al contrario que un mero objeto, hace referencia a otra cosa, a "algo"; "el arte es el tipo de cosas que dependen para su existencia de una teoría: sin teorías del arte, una pintura negra es sólo pintura negra y nada más" (Danto, 1981: 135). Precisamente, el primer rasgo que caracteriza al arte es su naturaleza representacional. Sin embargo, esto no es suficiente para caracterizar el arte como tal, ya que en principio todo tipo de representaciones, artísticas y no artísticas, cumplirían esta condición. Lo que distingue a las obras de arte de otras representaciones es el modo en el que las primeras son sobre su objeto; esto es, cumplirían la condición de "encarnar su significado". Una obra de arte es, por consiguiente, algo que encarna aquello que representa.

Así entonces, las diferencias ontológicas no se dirimen perceptivamente. Dado que la categoría de arte es una categoría ontológica, aquello que la define no pertenece al ámbito de lo perceptivo. Para el autor, entonces, la distinción entre una obra de arte de un objeto común no debe residir en una propiedad perceptiva. Por el contrario, necesita trazar allí una nueva propiedad relacional no perceptiva, para lo cual el autor propone la pertenencia a un "mundo del arte". Esto se ha entendido como un conjunto de individuos responsables de determinar los objetos que pueden ser considerados y evaluados artísticamente. La arbitrariedad de ello es evidente (y la sobre-teorización un poco pretenciosa, ciertamente). No obstante, esto no significa que ese conjunto de principios se sitúen fuera del objeto que se analiza: "el mundo del arte al que Danto atribuye 
un papel central en el problema de la demarcación, debe él mismo ser considerado un subproducto de las condiciones que determinan la naturaleza del arte en general” (Páez, 2008: I47). El término "mundo del arte" puede entenderse entonces como un concepto teórico, pero que denota principalmente una institución y un conjunto de prácticas sociales; "ver algo como arte requiere nada menos que esto, una atmósfera de teoría artística, un conocimiento de la historia del arte" (Danto, 198I: 135) Es decir, "un mundo del arte" (Danto, 1964).

La relación con esta atmósfera teórica que es constitutiva de un "mundo del arte" permite a Danto trazar la distinción entre unas cajas y otras. Pero, ¿en qué consiste esa atmósfera teórica? Como mencionamos, un "mundo del arte" puede entenderse como un conjunto de teorías o prácticas que determinan lo que se considera como arte en un determinado momento, así como el tipo de consideraciones valorativas acordes para juzgar una obra. Estaríamos aquí frente a otro gran problema, el de la relatividad de las interpretaciones y, creemos, también de la identificación de lo que denominamos "vestigio arqueológico", ya que ellos no responderían tampoco a ningún criterio preestablecido o absoluto. O meramente perceptivo si se prefiere.

\section{EL TERCER TIEMPO DEL OBJETO}

Llegados a este punto, podríamos plantear entonces que la vaga fisura entre los objetos comunes y los vestigios arqueológicos podría ser explicada dentro de un contexto cultural en el que las teorías determinaran los criterios de demarcación. Pero eso nos lleva a un problema mayor; esto es, el de la interpretación de los conjuntos materiales (Danto, 198I). En este punto, Danto considera que las interpretaciones transforman los objetos comunes en obras de arte. Es decir, la identificación artística es el mecanismo por el que transformamos objetos en obras de arte dotándolas de un carácter representacional. Es decir, la interpretación es la palanca con la que un objeto sale fuera del mundo físico y es situado en el "mundo del arte". Por lo tanto, es solamente en relación con una interpretación que un objeto es una obra de arte, lo que no significa que lo que se convierte en una obra de arte siga relacionada a lo anterior. En consecuencia, la obra de arte a la cual fue convertida el objeto común podría lograr una identidad propia, es decir, una nueva categoría,

"cada interpretación constituye una nueva obra, incluso si el objeto interpretado diferentemente permanece (...) invariable bajo la transformación. Un objeto o se convierte entonces en una obra de arte sólo bajo una interpretación I, donde $I$ es una especie de función que transforma o en una obra: I (o) $=\mathrm{W}$. Entonces incluso si o es una constante perceptiva, las variaciones en I constituye diferentes obras"5 (Danto, 198I: 125).

La teoría de interpretación es, por lo tanto, constitutiva. Para nosotros, eso quiere decir que un objeto es un vestigio únicamente en relación a una interpretación. $Y$ en ese sentido, la interpretación sería también transformadora. Esta transformación sería en síntesis nuestro tercer tiempo del objeto.

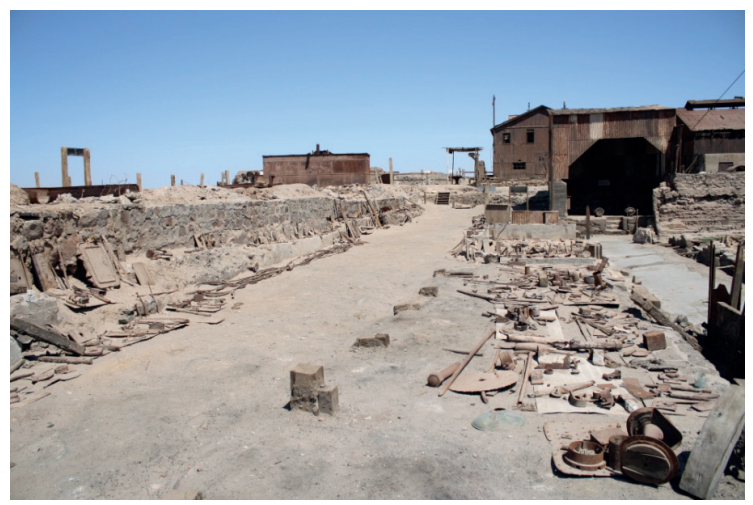

Figura 4: Exhibición de objetos, sector industrial de la oficina salitrera Santiago Humberstone, Región de Tarapacá, Chile (Foto: Rodrigo Lorca).

Figure 4: Exhibition of objects in the working area of industrial site Santiago Humberstone, Tarapacá Region, Chile (Photography: Rodrigo Lorca)

En un intento por redondear lo que ha sido propuesto, ejemplificaremos lo anterior con dos casos particulares que muestran las formas en las cuales la cultura material, en campos imaginarios propios, se representa para resaltar valores y significados (Lane, 2005). Tomaremos el caso del "mundo del salitre", como una analogía al "mundo del arte", ya que en él observamos dos espacios de exposición del objeto histórico que nos parecen sumamente interesantes: la primera corresponde a los resultados de un proyecto de rehabilitación de la zona industrial 
de la oficina salitrera Santiago Humberstone. Esta oficina salitrera se ubica en la Región de Tarapacá a $47 \mathrm{Km}$ de la ciudad de lquique y a 1050 m.s.n.m., en lo que se ha considerado históricamente como el principal y más rico yacimiento de caliche en el mundo. Debido a ello, el sitio se convirtió a fines del siglo $\mathrm{XIX}$ y principios del siglo $\mathrm{XX}$, en uno de los complejos urbano-industriales más importante de la zona y de Chile. Fundado en 1862 por la compañía Peruvian Nitrate Company con el nombre de Oficina La Palma, el lugar estará activo hasta 1929 cuando paraliza temporalmente sus faenas debido a la crisis económica mundial. No obstante, las labores se reanudarían posteriormente en 1933 con el nombre de Oficina Santiago Humberstone, aunque esta vez bajo la propiedad de la Compañía Salitrera de Tarapacá y Antofagasta (COSATAN), funcionando hasta 1959 cuando la sociedad definitivamente se disuelve. Finalmente, dada su importancia para la historia local y nacional, el sitio es incluido en el año 2002 en la lista de Sitios del Patrimonio Mundial de la UNESCO. En ese contexto, el año 2007 la Sociedad Química y Minera Chile (SQM) por medio de la Corporación Museo del Salitre (CMS) elaboraron un proyecto de intervención patrimonial, en el cual entre otros puntos, incluía la limpieza y ordenamiento de su zona industrial. Sin entrar en el detalle de sus resultados, una de las diversas actividades desarrolladas fue la habilitación de una ruta de recorrido peatonal, llamado sendero de interpretación, la cual fue utilizada para ubicar diversos objetos que hicieran recordar los procesos y los diferentes momentos que formaron parte de la extracción y elaboración del salitre (Figura 4).

El segundo ejemplo corresponde a la numerosa colección de objetos salitreros pertenecientes a un museo privado ubicado en la localidad de Baquedano. Este pequeño pueblo se ubica en lo que coloquialmente se conoce como "la mitad de la nada", en pleno desierto de Atacama a 90 kilómetros de la ciudad de Antofagasta (Región de Antofagasta). Fundado en 1910 como una estación de trenes, ha formado parte desde entonces de la red ferroviaria que une el altiplano boliviano con la costa del Pacífico. Hoy, además de servir como punto neurálgico del transporte de mineral, mantiene una pequeña población dependiente de la comuna de Sierra Gorda y del constante tráfico de trabajadores mineros de las grandes cupríferas instalada en la zona. Es sabido que los habitantes de estas localidades desérticas se sienten especialmente orgullosos del pasado salitrero de la región, por lo que es común encontrar colecciones privadas de objetos provenientes de las antiguas oficinas salitreras que funcionaron desde fines del siglo XIX hasta mediados del siglo $X X$. En ese espíritu, fue creado hace varios años atrás un pequeño museo local y privado, con una extraordinaria cantidad de objetos recuperados de estos lugares (Figura 5): artículos de mesa, herramientas de trabajo, papelería o fichas, todo lo imaginable puede ser encontrado allí. No obstante, más allá de su riqueza material, lo que nos interesa de ambos ejemplos es la manera en la cual los objetos fueron descontextualizados y colocados en una exhibición sin orden aparente ni guión museológico, pero cuyo propósito fue la de conservarlos haciendo partícipe a los visitantes a una experiencia en la cual el pasado es revivido a través de la materialidad del pasado reciente. Como se puede ver para el caso de Humberstone en la Figura 4, los mismos objetos que antes se encontraban acumulados en grandes depósitos de desechos industriales como basura contemporánea, fueron desde aquél momento separados, limpiados y exhibidos en el mismo espacio laboral del sitio.

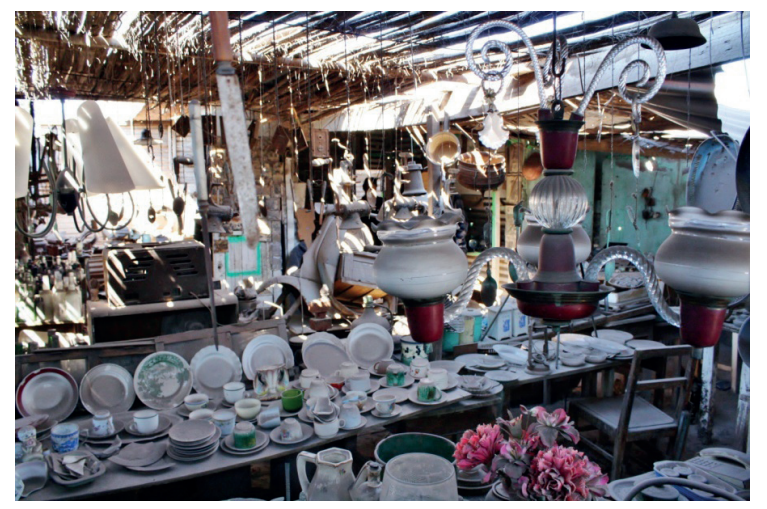

Figura 5: Exhibición de colección de objetos en museo privado, Baquedano, Región de Antofagasta, Chile (Foto: Rodrigo Lorca).

Figure 5: Exhibition of objects collection in a private museum, Baquedano, Antofagasta Region, Chile (Photography: Rodrigo Lorca).

Con estos dos ejemplos queremos ilustrar también los diferentes tiempos de la materialidad a través de dos similares modalidades de exhibición, que sirven para poner en perspectiva lo que proponemos. Hasta aquí hemos relacionado las representaciones de la materialidad en la literatura, y discutido una 
forma en la cual los objetos se transforman mediante una constitución interpretativa en una obra de arte. Si mencionamos un tercer tiempo, es porque buscamos en la ficción del objeto arqueológico, tal como se presenta en los ejemplos señalados arriba, pensar otra forma de este tipo de relación entre el individuo y la materialidad desde la constitución interpretativa arqueológica. Mientras los ready-made imponen en el arte esta nueva visión del objeto autoreproducible y des-territorializado conceptualmente, el objeto arqueológico-patrimonial por su parte, central en esta configuración, coloca en primer plano el problema del tiempo arqueológico, que de aquí en adelante creemos que se vuelve esencial. Como lo hemos ejemplificado a través de los casos de Humberstone y el museo privado de Baquedano, observamos que a aquellos sistemas de familiaridad de los objetos, ya sea el objeto común, la obra de arte $\circ$ el vestigio arqueológico, estará asociado lo que podríamos llamar los "sistemas de temporalidad" (Baudrillard, 1968), los cuales nos permiten reconsiderar las cronologías de la materialidad para así lograr establecer los criterios que permitirían conservarla. En decir, las categorías reemplazan el objeto con el fin de insertarlos en otro tipo de dinámicas temporales. En ese sentido, si exploramos las formas de des-territorialización de los objetos como forma de desarraigo de la rigidez de los sistemas de clasificación y sus categorías, comenzamos también a recorrer espacios de límites muy difusos en los cuales las configuraciones materiales se vuelven difíciles de identificar: ¿Es arqueológico? ¿Es patrimonial? En este artículo es lo que hemos propuesto identificar como el tercer tiempo del objeto, entendiendo que "esencialmente, descubrir el pasado es tomar conciencia de cuan variado es el presente" (Olivier, 2004: 207).

Como corolario debemos plantear por lo tanto, desde una mirada arqueológica, la siguiente pregunta: ¿Cuál podría ser entonces el rol de la literatura como fuente de datos frente al rol desmitificador de la historia y sus documentos oficiales? Hemos visto que la ficción literaria, aunque sumamente descriptiva, pierde peso frente a la objetividad científica de la historia oficial (Hennion y Latour, 1993). El objeto histórico de valor científico se desvincula entonces del objeto artístico emanado de la ficción, por lo que nos vemos consecuentemente obligados a entender el carácter inestable de los procesos por el cual el objeto a cruzado para poder llegar hasta nosotros aquí y ahora. No obstante, esta inestabilidad nos permite también comprender que el objeto arqueológico que hoy llamamos "vestigio" es el fruto de un largo proceso de transformación mediatizada por la interpretación. En ese sentido, la conservación patrimonial estrechamente ligada a este proceso de transformación, cuyos fines diversos por lo general de carácter académico y científico, también extrae al objeto de su tiempo cronológico para ser insertado en un nuevo tiempo arqueológico, el cual además seguirá trabajando su propia materia, desde ahora en otro "tipo" de tiempo (Oliver, 2008). Así como el enfoque patrimonial y la preservación de los bienes culturales fomentan la exhibición y conservación de la materialidad en museos, exposiciones $\mathrm{o}$ instituciones culturales, el objeto cotidiano o industrial, considerado reproducible, se presenta por lo tanto de ahora en adelante como un objeto artístico y patrimonial, único e irrepetible; una "cosa" que es más que un mero objeto (Bazin y Bensa, 1994). El objeto igualmente arqueológico, igualmente ficcionado (Figuras 4 y 5 ).

\section{CONSIDERACIONES FINALES}

En esta breve discusión hemos querido exponer la idea de que simultáneamente a un movimiento literario que sitúa y destaca los objetos, alimentado además desde fines del siglo XIX por los avances tecnológicos del mundo científico e industrial, se observa también un proceso de desintegración de los límites conceptuales de la "obra de arte" y luego del "vestigio arqueológico". Hemos visto que lo anterior crea inevitablemente una nueva relación entre referentes y atributos, y finalmente entre objeto e individuo. El rol de la materialidad histórica se convierte desde entonces en un tema de investigación para la literatura (Brown, 2003, 2004, 2010; Caraion, 2005, 2007), luego en un problema de definiciones para el arte (Carroll, 1995; Danto, 1964, 1981) y, finalmente como resultado de lo anterior, en un interesante marco de análisis para la arqueología (Olivier, 2008).

Creemos que considerar las materialidades como prácticas sociales derivadas de esta relación, es entender también que estas prácticas reenvían hacia actitudes en situaciones específicas; en un "sistema 
hablado" propio y concreto (Baudrillard, 1968). La representación de los objetos en la literatura reflejan las percepciones de una sociedad frente a los cambios de su contexto físico, la exhibición del objeto nos muestra los límites difusos entre objeto común y obra de arte, $y$ finalmente el objeto patrimonial de la arqueología, permite entrever el rol del vestigio arqueológico y el problema que la materialidad del pasado reciente presenta a la interpretación y sus efectos en el presente. De lo anterior, es claro que los tiempos del objeto no son en ningún caso lineales ni mucho menos secuenciales; son en cambio el resultado de una serie de imbricaciones y transformaciones, muchas veces generadas inconscientemente.

En síntesis, hemos querido proponer la idea de que en una relación de simetría entre la materialidad de ficción y el vestigio arqueológico, se superponen e imbrican problemas de interesante perspectiva de análisis. Aunque esbozado sólo de forma preliminar nos ha interesado también formular como problema de estudio, en el marco de una lectura arqueológica alternativa sobre los tiempos del objeto, las modalidades de descripción de ellos desde el sentido de ésta como voz narrativa y estructura del discurso tanto de ficción como académico (texto) (Holtorf, 20l0), el lugar de los objetos en la sociedad que los describe (contexto) y su rol específico como protagonistas y no solamente como accesorios (agentes). Queda pendiente aún profundizar, entre otras cosas, la reflexión sobre el valor de cada uno de los tiempos del objeto: el valor simbólico del objeto literario, el valor estético del objeto artístico y el valor patrimonial del objeto arqueológico (véase por ejemplo Shanks y Tilley, 1987; Graeber, 200 I; Samuels, 2008; Papadopoulos y Urton, 2012), así como una evaluación crítica de las innovaciones tecnológicas en multimedia, la realidad virtual y los nuevas modalidades de registros visuales, tales como la documentación en 3D, que demuestra notras formas de relacionarnos con la materialidad en el presente (Olsen et al., 20I2). Por último, creemos que lo propuesto aquí entrega un aspecto interesante para una discusión amplia sobre el uso social del objeto histórico y del pasado reciente en la práctica arqueológica-histórica asociada a las políticas económicas y patrimoniales en esta nueva "expansión capitalista" del siglo XXI (Cáceres,
1999; Patterson, 1999; Cáceres y Westfall, 2004; Clifford, 2004; Dawdy, 2009; Pétursdóttir, 2013). En resumen, hemos querido abrir una discusión sobre el tiempo arqueológico considerando que es también plausible una lectura de los objetos no solamente como vestigios físicos sino también como representaciones. Esto último con especial atención al carácter transformador de la relación existente al momento en el cual los objetos formaron parte en el pasado, con el instante en el cual son leídos, interpretados y conservados en el presente.

Si bien proponemos estos tres tiempos del objeto como marcos de análisis, no estamos convencidos por el contrario que estos sean los únicos. Nuestra aproximación no pretende discutir el detalle de lo objetivo o el dato material frente a la fuente documental, sino que las actitudes $y$ emociones asociadas al recuerdo a través de las representaciones de la materialidad, en el cual pueda finalmente entenderse que no trabajamos con un tiempo cronológico lineal, sino que uno arqueológico multidimensional. Como bien lo plantea Olivier: "no debemos olvidar que la arqueología no es una disciplina histórica estándar; ella trata con la memoria registrada en la materia y no con eventos - momentos del pasado" (Olivier, 2004: 209). En definitiva, buscamos desde el imaginario de los objetos una experiencia de la memoria (Pétursdóttir, 2012) que evite "la ilusión moderna de la linealidad" (Castoriadis, 1975). Lo anterior se resume de manera perfecta en los siguientes versos de Teiller (1992:45),

"Pues lo que importa no es la luz que encendemos día a día / sino la que alguna vez apagamos / para guardar la memoria secreta de la luz. / Lo que importa no es la casa de todos los días / sino aquella oculta en un recodo de los sueños. / Lo que importa no es el carruaje I sino sus huellas descubiertas por azar en el barro. / Lo que importa no es la lluvia / sino sus recuerdos tras los ventanales del pleno verano".

Agradecimientos: a Yerko Araneda por su apoyo bibliográfico. A Patricia Roldán y el Museo de Artes Decorativas por su buena disposición y recibimiento para fotografiar la colección. A los editores y evaluadores, cuyos comentarios y sugerencias nos hicieron ver aspectos interesantes para mejorar el artículo. 


\section{NOTAS}

I Para ilustrar la amplitud del tema, basta con recordar las célebres excavaciones realizadas por Heinrich Schliemann (I822-1890) en base a los versos de la llíada, en su afán por encontrar la Troya descrita por Homero.

2 Concordamos aquí con los acertados comentarios de los evaluadores, quienes nos recuerdan que la nomenclatura utilizada en el pasado son las mismas que se utilizan hoy para las tipologías materiales históricas.

3 Utilizaremos el término "vestigio" para referirnos a la materialidad categorizada como "resto arqueológico".

4 En este artículo incluimos referencias de las obras de los escritores chilenos Alberto Blest Gana (1830-1920), Moisés Vargas (1842-1898), Luis Orrego Luco (I866-1948), Baldomero Lillo (1867-1923), junto con el escritor uruguayo Eduardo Galeano (1940).

\section{BIBLIOGRAFÍA}

Alegría Licuime, L. 2007. "Las colecciones del Museo Histórico Nacional de Chile: ¿"Invención” ○ "construcción" patrimonial?". Anales del Museo de América I5: 237-248. Paris.

Baudrillard, J. 1968. Le Système des Objets. Gallimard,

----- 2009 [1974]). La Sociedad de Consumo. Sus Mitos, sus Estructuras. Siglo XXI, Madrid.

Bazin, J., y A. Bensa.1994. "Les objets et les choses. Des objets à “la chose'”. Genèses 17: 4-7.

Blest Gana, A.1904. Los Transplantados. Tomo Segundo. Garnier Hermanos Libreros Editores, Paris.

Bourdieu, P. 1998. La Distinción. Criterio y Bases Sociales del Gusto. Taurus, España.

Brown, B. 2003. A Sense of Things: The Object Matter of American Literature. University of Chicago Press, Chicago.

---- 2004. Things. University of Chicago Press, Chicago.

---- 2010. "Objects, Others, and Us (The Refabrication of Things)”.Critical Inquiry 36(2): I83-217.

Buchli, V y G. Lucas. 200I. Archaeologies of the Contemporary Past. Routledge, New York.

Cáceres, I. 1999.“Arqueología y Sistema de Evaluación de Impacto Ambiental”. Boletín de la Sociedad Chilena de Arqueología 28: 47-54.

Cáceres, I. y C. Westfall. 2004. "Trampas y amarras: ¿es posible hacer arqueología en el Sistema de Evaluación de Impacto Ambiental?". Chungará 36: 483-488.

Caraion, M. 2005. "Objets en liberté: réflexions préliminaires”. En: Objets en liberté, editado por M. Caraion, pp. 7-27. Archipel, Lausanne.

---- 2007. “Objets en littérature au XIXe siècle”. Images Re-vues 4: 2-17.

Carroll, N. 1995. “Danto, Style, and Intention”. The Journal of Aesthetics and Art Criticism 53 (3): 25 I-257.

Castoriadis, C. 1975. L'Institution Imaginaire de la Société. Editions du Seuil, Paris.

Clifford, J. 2004.“Looking SeveralWays:Anthropology and
Native Heritage in Alaska”.Current Anthropology 45(I): 5-30.

Danto, A. 1964. “The Artworld”. The Journal of Philosophy $6 I$ (I9): 57I-584.

---- 198I.The Transfiguration of the Common place. Harvard University Press, Cambridge, Massachusetts.

Dawdy S.L. 2009. "Millennial archaeology. Locating the discipline in the age of insecurity". Archaeological Dialogues I6(2): |3|-| 42.

De Rokha, P. 1954. "Fuego Negro. III Lamento en Piedra”. En: Antología 1916-1953, pp. 499-5 I0. Multitud, Santiago.

Dickie, G. 1969.“Defining Art”. American Philosophical Quarterly6 (3): 253-256.

Faba, P. 2013. "Cultura visual y memoria en el Chile del siglo XIX. Redefiniendo el Coloniaje a través de su exhibición”. Revista de Teoría del Arte 24: 13-33.

Galeano, E.1984. Memorias del Fuego II: Las Caras y las Máscaras. Siglo XXI, Madrid.

González-Ruibal, A. 2006. "The past is tomorrow. Towards an archaeology of the vanishing present". Norwegian Archaeological Review 39(2): I I0-I 25.

Graeber, D. 200I. Towards an Anthropological Theory of Value: the False Coin of our Dreams, Palgrave, New York.

Habermas, J.1984. The Theory of Communicative Action. Beacon Press, Boston.

Harrison, R. y J. Schofield. 2010. After Modernity. Archaeological Approaches to the Contemporary Past. Oxford University Press, Oxford.

Heinich, N. 1993.“'Les objets-personnes: fétiches, reliques et œuvres d'art”. Sociologie de l'art $6: 25-55$.

----- 2009. La Fabrique du Patrimoine. De la Cathédrale à la Petite Cuillère. Maisondes Sciences de l'homme, Paris.

Hennion, A. y B. Latour. 1993. “Objet d'art, objet de science. Note sur les limites de l'anti-fétichisme". Sociologie de l'art: 7-24.

Holtorf, C. 2010. "Meta-stories of archaeology". World Archaeology 42(3): 38I-393.

Lane, P. 2005. "The material culture of memory". En: The qualities of time. Anthropological approaches, editado por W. James y D. Mills, pp. 19-34. Berg, Oxford-New York.

Lillo, B. 1968. “El alma de la máquina”. En: Obras completas, pp. 262-264. Nascimento, Chile.

Marx, K. 1980. El Capital. Crítica de la Economía Política.Vol. I.Siglo XXI, Madrid.

Olivier, L. 2004. "The past of the present. Archaeological memory and time”. Archaeological Dialogues I0(2): 204-2I 3. Seuil, Paris.

----- 2008. Le Sombre Abîme du Temps:Mémoire et Archéologie.

Olsen, B. 2013. In Defense of Things. Archaeology and the Ontology of Objects. Altamira Press, UK.

Olsen, B., M. Shanks, T. Webmoor y C. Witmore. 2012. Archaeology. The Discipline of Things. University of California Press, Berkeley \& Los Angeles.

Orrego Luco, L. 1908. Casa Grande. Escenas de la vida en Chile. Zig-Zag, Chile. 
Orser, C.E. 2000. Introducción a la Arqueología Histórica. Asociación Amigos del INAPL, Buenos Aires.

Páez, A. 2008. "El problema de la demarcación en estética: una crítica del criterio de Danto". Revista de Estudios Sociales 29:|46-I55.

Papadopoulos, J. y G.Urton (eds.). 2012. The Construction of Value in the Ancient World. Cotsen Institute of Archaeology, University of California, Los Angeles.

Patterson, T. 1999. "The political economy of archaeology in the United States". Annual Review of Anthropology 28:I55-I74.

Pétursdóttir, P. 2012. "Small things forgotten now included, or what else do things deserve?". International Journal of Historical Archaeology 16(3): 577-603.

--12013. "Concrete matters: Ruins of modernity and the things called heritage". Journal of Social Archaeology I3(I): 3I-53.

Perec, G. 1965. Les Choses. René Julliard, Paris.

Rivera, F. y A. Delgado. 2009. Informe análisis de vidrio, sitio El Churque 10. Proyecto hipógeno Compañía Minera El Carmen de Andacollo. Manuscrito en posesión de los autores.

Samuels, K.L. 2008. "Value and significance in archaeology". Archaeological Dialogues I5(SII):7I-97.

Schávelzon, D. 2002. The Historical Archaeology of Buenos Aires. A City at the End of the World. Kluwer Academic Publishers, New York, Boston, Dordrecht, London, Moscow

Shanks, M. y C. Tilley. 1987. Re-Constructing Archaeology. Theory and Practice. Cambridge UniversityPress, Cambridge.

Teiller, J. 1992. Los Dominios Perdidos. Fondo de Cultura Económica, Chile.

Vargas, M. 1870. Adiós a la Vida. Imprenta de la República, Santiago.

Vicuña Mackenna, B. 1873. Catálogo Razonado de la Exposición del Coloniaje. Celebrada en Santiago de Chile en septiembre de 1873. Por uno de sus miembros de la Comisión Directiva. Imprenta Sud-América, De claro y Salinas, Santiago. 